\title{
Model disability survey: a pilot study in the North Batinah governorate
}

\author{
Adhra Al-Mawali, ${ }^{, *}$ Magdi Morsi, ${ }^{a}$ Hilal Al-Kharusi, ${ }^{a}$ Waleed Al-Shekaili, ${ }^{a}$ Carla Sabariego, ${ }^{b}$ and Lindsay Lee ${ }^{c}$
}

\author{
${ }^{a}$ Centre of Studies \& Research, Ministry of Health, Muscat, Sultanate of Oman. \\ bHealth Systems, Policy \& Communication Unit, Disability Policy Group, Swiss Paraplegic Centre, Nottwil, Switzerland. \\ 'Blindness and Deafness Prevention, Disability and Rehabilitation Unit, World Health Organization, Geneva, Switzerland. \\ *Correspondence to Adhra Al-Mawali (email: adhra.almawali@gmail.com). \\ (Submitted: 14 August 2018 - Revised version received: 23 September 2018 - Accepted: 29 September 2018 - Published online: 26 December 2018)
}

\begin{abstract}
Objective The continuous shift to non-communicable diseases in Oman require a more consistent inclusion of functioning and disability information in health monitoring and evaluation frameworks. Oman currently relies on the census to generate data on disability which is usually very limited in scope, and provides only rough prevalence estimates of persons with specific impairments. The lack of accurate, comprehensive and up-to-date information on disability is a major obstacle for planning services and allocating resources. Thus, there was a need to conduct a comprehensive population-based survey to fill this information gap. The pilot aimed to examine the feasibility of the Arabic version of the Model Disability Survey (MDS) in the cultural context of Oman, identify potential problems with the survey, and develop strategies to deal with them before a large national implementation is launched.

Methods The MDS is the tool recommended by the World Health Organization(WHO) to collect comprehensive data about functioning and disability to quantify both the impact of health conditions or impairments as well as the impact of the environment on disability. To determine the applicability of measuring disability, a pilot study was carried out in the North Batinah governorate of Oman with a convenience sample of 288 adults aged 18 year or older in collaboration with WHO.

Results The overall disability prevalence was estimated to be $14.6 \%(n=42)$ in the MDS pilot study calculated by determining the proportion of people in the population who experience severe performance problems. The overall results corroborated that the Arabic translation of the MDS survey tool works well in the field and is suitable for a large scale implementation, after minor revisions.

Conclusion Since the results of this pilot study in Oman show that the disability measurement survey tool has been successfully tested, we recommend that the MDS be extended and implemented nationally. It should also be integrated to existing routine household surveys to allow continuous monitoring of disability in countries.

Keywords disability, physical functioning, cognitive functioning, disability survey, oman
\end{abstract}

\section{Introduction}

Oman is facing a continuous rise in non-communicable diseases (NCDs). NCDs - including hypertension, heart disease, asthma, diabetes, stroke, depression, back pain and hearing and vision disorders - are frequently associated with moderate to high levels of disability. ${ }^{1}$ Additionally, life expectancy is steadily increasing due to improvements in health care, and ageing is also associated with disability. Increased life expectancy and the high prevalence of NCDs require a more consistent inclusion of functioning and disability information in health monitoring and evaluation frameworks. In recognition of its importance for complementing information on mortality and morbidity, the International Classification of Diseases, Eleventh Revision will include information on functioning and disability. ${ }^{2}$

Information at population level collected through a dedicated disability survey is relevant for evaluating the effect of public health interventions targeting functioning and disability. Such interventions can be directed at improving or optimizing functioning of individuals, for instance through provision of rehabilitation services, or at reducing environmental barriers, for instance through dedicated transportation, employment or accessible health care policies. Information on functioning and disability is also relevant for developing evidence-based plans of action and policies, for planning and allocating resources to health and social services, and to monitor and report on the implementation of the convention on the rights of persons with disabilities and the indicators of the sustainable development goals.
In the WHO International Classification of Functioning, Disability and Health (ICF), disability encompasses impairments, such as problems with breathing, memory or pain, limitations of activities, such as problems with mobility and self-care, and participation restrictions, such as problems at work or school. Although associated with health conditions, disability is not understood as the direct consequence of a disease. In line with the ICF, disability is the outcome of the interaction between a health condition (e.g. arthritis, back problems, hearing loss or depression) and environmental barriers (e.g. limited access to health care, negative attitudes of others, inaccessible transportation and public buildings, or lack of inclusive employment laws). ${ }^{3}$ Disability is also understood in the ICF as a continuum, ranging from no disability (or full functioning) to very high levels of disability. Disability is therefore a matter of degree and universal, since any person with health conditions or age-related decrements in health will experience disability to some extent at some point in his or her life. ${ }^{3}$ Patterns and the level of disability in countries are influenced therefore by trends in health conditions and by trends in environmental barriers and other factors - such as road traffic crashes and accidents. ${ }^{4}$

The Sultanate of Oman relies currently on the census to generate data on disability. Based on the census in 1993, the disability rate was $1.9 \%$ among Omani nationals. This rate rose to $2.4 \%$ in 2003 and to $3.2 \%$ in $2010 .^{5,6}$ Such increase could be partially attributed to an actual increase chronic health conditions associated with disability coupled with 
better care resulting in higher life expectancy. However, census data is usually very limited in scope, and provides only rough prevalence estimates of persons with specific impairments. The lack of accurate, comprehensive and up-to-date information on disability is a major obstacle for planning services and allocating resources for the care and rehabilitation of persons with different levels of disability. Thus, there is a need to conduct a comprehensive population-based survey to fill this information gap.

\section{Choosing the Model Disability Survey Tool to Measure Disability in Oman}

The model disability survey (MDS) was developed by the World Bank and WHO in 2012 and is the tool recommended by WHO to collect comprehensive data about functioning and disability, in line with the ICF. The MDS is implemented to estimate the distribution of disability in a country as well as how many people have severe, moderate and mild disability. The MDS also identifies their unmet needs as well as the barriers and inequalities faced by these persons in daily life. Thus, data generated by the MDS is being used by countries to quantify both the effect of health conditions or impairments as well as the effect of the environment on disability. This allows countries to determine which interventions and policies will likely produce the most benefit for the population.

To determine the applicability of measuring disability, a pilot study was carried out in Oman by the Ministry of Health in 2016, in collaboration with WHO. The pilot aimed to examine the feasibility of the Arabic version of the MDS in the cultural context of Oman; identify potential problems with the survey, and; develop strategies to deal with them before a large national implementation is launched.

\section{Methodology}

A convenience sample of 288 adults aged 18 year or older was selected in this pilot study. The selection was done to include men and women of varied ages as well as to include both healthy respondents and persons with impairments and health conditions. It is to be noted that the sample used is not representative of the Omani population. Data collection was done in the morning only and the individual who was at home during the visit was selected for the interview. For this reason, the sample includes more women, who were not working or not studying. Their socio-demographic characteristics are shown in Table 1.

A household questionnaire was used which was answered by the head of the household and included a household roster and a children module. The individual questionnaire was answered by a randomly selected adult member of the household. The questionnaire contained eight modules: socio-demographic characteristics; work history and benefits; environmental factors; functioning; health conditions and capacity; health care utilization; well-being; and empowerment. ${ }^{8}$

\section{Results and Discussion}

The overall disability prevalence estimated in the MDS is calculated by determining the proportion of people in the population who experience severe performance problems. For the

\begin{tabular}{lcc} 
Table 1. $\begin{array}{l}\text { Demographic characteristics of sample used in the } \\
\text { pilot study }\end{array}$ & \multicolumn{2}{c}{ MDS sample } \\
\hline \multicolumn{1}{c}{ Characteristic } & N & Percentage (\%) \\
\cline { 2 - 3 } & 102 & 35.4 \\
\hline Gender & 186 & 64.6 \\
Male & 288 & 100 \\
Female & & \\
Total & 150 & 52.1 \\
Age (years) & 96 & 33.3 \\
18-39 & 42 & 14.6 \\
40-59 & 288 & 100 \\
>60 & & \\
Total & 48 & 16.7 \\
Marital status & 219 & 76 \\
Single & 4 & 1.4 \\
Married & 17 & 5.9 \\
Separated or divorced & 288 & 100 \\
Widowed & & \\
Total & 48 & \\
\hline
\end{tabular}

present pilot study this rate is $14.6 \%(N=42)$. Approximately $21 \%$ of included households had a person in need of extra support for health care and $18 \%$ had at least a person in need of extra physical or emotional care or support.

Persons experiencing severe capacity difficulties are generally at high risk of experiencing severe performance problems if no accommodations are in place. It is therefore of interest to know the percentage of persons experiencing severe capacity difficulties and severe performance problems, which in the case of this pilot study are $14.2 \%$ of the whole sample. However, in this sample 3.1\% experience severe capacity difficulties but do not experience severe performance problems, suggesting that the environment is facilitating to some extent for these individuals. The proportion of persons in the sample experiencing severe performance problems without severe capacity difficulties is very low: $0.3 \%$. Table 2 presents the socio-demographic characteristics of these three distinct groups. It is important to note, however, that the sample size is too small to allow for interpretations.

Capacity is the synthesis of all of the intrinsic physical and mental capacities of a person, determined by their health state. One of the most important objectives for carrying out the pilot study in Oman is to confirm that metric scales of capacity and performance can be created for the country using item response theory. The higher the scores are, the worse the difficulties in capacity. Figure 1 shows the distribution of the convenience sample on the capacity continuum; the scale ranges from 0 no capacity difficulties to 100 (very severe capacity difficulties). The MDS enables decision-makers to go beyond to identify the factors that are responsible for inequalities which allows them to identify appropriate and effective interventions and policies, whilst also distribute outcomes of interest along the disability continuum for those who experience severe, moderate and mild disability, as identified by suitable thresholds. 
Table 2. Demographic characteristics of disability groups

Severe disability

\begin{tabular}{|c|c|c|c|c|c|c|}
\hline \multirow{3}{*}{ Characteristic } & \multicolumn{6}{|c|}{ Severe disability } \\
\hline & \multicolumn{2}{|c|}{$\begin{array}{l}\text { Group 1: performance is better } \\
\text { than capacity }(N=9)\end{array}$} & \multicolumn{2}{|c|}{$\begin{array}{c}\text { Group 2: performance and capacity } \\
\text { equal }(N=41)\end{array}$} & \multicolumn{2}{|c|}{$\begin{array}{l}\text { Group 3: capacity is better than } \\
\text { performance }(N=1)\end{array}$} \\
\hline & $N$ & Percentage (\%) & $N$ & Percentage (\%) & $N$ & Percentage (\%) \\
\hline \multicolumn{7}{|l|}{ Gender } \\
\hline Male & 3 & 33.3 & 14 & 34.1 & 1 & 100 \\
\hline Female & 6 & 66.7 & 27 & 65.9 & 0 & 0 \\
\hline \multicolumn{7}{|l|}{ Age group (years) } \\
\hline $18-39$ & 3 & 33.3 & 13 & 31.7 & 0 & 0 \\
\hline $40-59$ & 5 & 55.6 & 10 & 24.4 & 0 & 0 \\
\hline$>60$ & 1 & 11.1 & 18 & 43.9 & 1 & 100 \\
\hline \multicolumn{7}{|l|}{ Work status } \\
\hline Not working & 0 & 0 & 4 & 66.7 & 1 & 100 \\
\hline Working & 2 & 100 & 2 & 33.3 & 0 & 0 \\
\hline \multicolumn{7}{|l|}{ Education } \\
\hline No education, illiterate & 4 & 44.4 & 31 & 75.6 & 1 & 100 \\
\hline No education, literate & 0 & 0 & 1 & 2.4 & 0 & 0 \\
\hline Less than primary school & 1 & 11.1 & 4 & 9.8 & 0 & 0 \\
\hline Prep school not completed & 1 & 11.1 & 0 & 0 & 0 & 0 \\
\hline Prep school & 2 & 22.2 & 3 & 7.3 & 0 & 0 \\
\hline Secondary & 1 & 11.1 & 2 & 4.9 & 0 & 0 \\
\hline Higher & 0 & 0 & 0 & 0 & 0 & 0 \\
\hline \multicolumn{7}{|l|}{ Marital status } \\
\hline Single & 0 & 0 & 13 & 31.7 & 0 & 0 \\
\hline Married or legal cohabitation & 8 & 88.9 & 20 & 48.8 & 1 & 100 \\
\hline Separated or divorced & 0 & 0 & 1 & 2.4 & 0 & 0 \\
\hline Widowed & 1 & 11.1 & 7 & 17.1 & 0 & 0 \\
\hline
\end{tabular}

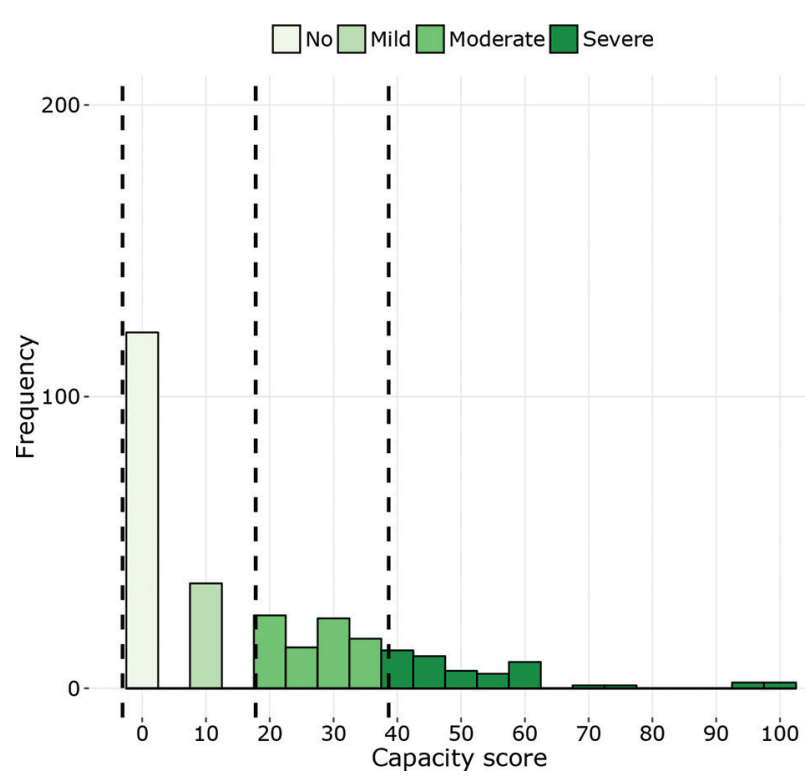

Fig. 1 Capacity continuum and distribution of the population on the continuum.
In several countries, the proportion of severe capacity difficulties is analogous to previous prevalence estimates of disability calculated from the census; however, in the MDS this proportion is usually much higher than previous estimates. In the case of this pilot study, the severe capacity difficulties proportion is $17.4 \%$ of the included population. This larger estimate is mostly due to the broader definition of disability used in MDS. Historically, censuses identify people with a narrow set of conditions or impairments as "disabled", while in the MDS the capacity estimate is a composite score of difficulties in 17 functioning domains. In this pilot sample, $27.8 \%$ experience moderate capacity difficulties while $12.5 \%$ experience mild capacity difficulties.

Interviewers rated that approximately $96 \%$ of respondents were highly or very highly cooperative. Additionally, the answers of approximately $96 \%$ of the respondents were rated as highly or very highly accurate by interviewers.

Regarding the questions, following problems were observed. Questions such as 'How many weeks do you work during the year?' were perceived as difficult due to problems to calculate the number of weeks accurately. Questions about attitudes of others posed some problems as the majority of 
respondents asked about the relationship of these questions to health and disability. Especially questions such as 'Do people around you tend to become impatient with you?' and 'Is living with dignity a problem for you because of the attitudes and actions of others?' caused problems. The introduction of the "attitudes of others" questions would need a revision that stresses why these questions are relevant. Finally, question 'To what extent would you agree with the statement that you are a person who tends to find fault with others?' was perceived as embarrassing by respondents, and questions 'Do you think that the problems you have told me about have made you a stronger person? \& Do you think that the problems you have told me about have made you more determined to reach your goals?' would need a response option "does not have any problem" for respondents who reported having no problems at all. These issues need revisions before a full implementation can be started.

\section{Conclusion}

Our overall results above corroborate that the Arabic translation of the MDS survey tool works well in the field and is suitable for a large scale implementation, after minor revisions. It is recommended by WHO that a stand-alone version of the MDS is implemented every $5-10$ years while a brief version - a module developed to be integrated to existing household surveys - should be implemented routinely to allow a continuous monitoring of disability in countries. ${ }^{10}$ Since the results of this pilot study in Oman show that the disability measurement survey tool has been successfully tested, we recommend that this is extended and implemented nationally.

\section{Conflict of Interest}

None.

\section{References}

1. Al-Mawali A. Non-communicable diseases: shining a light on cardiovascular disease, Oman's biggest killer. Oman Med J. 2015;30:227-228.

2. Escorpizo R, Kostanjsek N, Kennedy C, Nicol MM, Stucki G, Ustün TB. Functioning Topic Advisory Group (fTAG) of the ICD-11 Revision: Harmonizing WHO's International Classification of Diseases (ICD) and International Classification of Functioning, Disability and Health (ICF): importance and methods to link disease and functioning. BMC Public Health. 2013;13:742.

3. World Health Organization. International Classification of Functioning, Disability and Health (ICF). 2018. Available from: http://www.who.int/ classifications/icf/en/ [cited 2018 September 5].

4. World Health Organization. WHO Global Disability Action Plan 2014-2021: Better Health for all People with Disability. World Health Organization, Geneva, 2015.

5. Ministry of National Economy Oman. Disability Statistics in the Sultanate of Oman: The Experience of Data Collection During 3 Censuses $(1993,2003,2010) .11^{\text {th }}$ Meeting of the Washington Group on Disability Statistics. Southampton, Bermuda. 2011. Available from: https://

www.cdc.gov/nchs/ppt/citygroup/meeting11/wg11_session6_2_aldagheishi.pdf)

6. Al-Balushi T, Al-Badi AH, Ali S. Prevalence of disability in Oman: statistics and challenges. Can J Appl Sci. 2011;1:81-96.

7. World Health Organization. Model Disability Survey: Providing Evidence for Accountability and Decision-making. Available from: http://www.who.int/ disabilities/data/mds_v4.pdf?ua=1 [cited 2018 September 6].

8. World Health Organization. Model Disability Survey. [cited 2018 September 6]; Available from: http://www.who.int/disabilities/data/model-disability-survey4 pdf?ua=1.

9. Cieza A, Sabariego C, Bickenbach J, Chatterji S. Rethinking disability. BMC Med. 2018;16:14.

10. Kostanjsek N. Use of The International Classification of Functioning, Disability and Health (ICF) as a conceptual framework and common language for disability statistics and health information systems. BMC Public Health. 2011;11:S3 (BioMed Central). 